\title{
On Crossley's contribution to the development of graph based algorithms for the analysis of mechanisms and gear trains
}

\author{
Ettore Pennestrì ${ }^{\mathrm{a}, *}$, Nicola Pio Belfiore ${ }^{\mathrm{b}}$ \\ a University of Rome Tor Vergata, Dept. of Enterprise Engineering, Via del Politecnico, 1, 00133 Rome, Italy \\ b Sapienza University of Rome, Dept. of Mechanical and Aerospace Engineering, Via Eudossiana, 18, 00149 Rome, Italy
}

\section{A R T I C L E I N F O}

\section{Keywords:}

Crossley

Graph Theory

Mechanisms

Gear trains

Kinematics

Dynamics

\begin{abstract}
A B S T R A C T
This paper celebrates a particular branch of Crossley's early work dedicated to Mechanism Science, which deals with a rigorous introduction of Graph Theory to the study of some fundamental and intrinsic properties of kinematic chains and mechanisms. Although such idea gave its main outcome in Type and Number Synthesis (which has been much better and extensively described in another paper of the present special issue) some other intriguing side effects appeared, later in Mechanism Science, which yielded several results, and are still in the center of research and industrial world interest, such as, to name but a few, the automatic generation of the equations governing kinematic, static force and dynamic analysis of mechanisms and geared trains, the power flow analysis, the computation of the efficiency and, finally, the never fully explored structure-to-function mapping, which the present contribution points out to be still a challenge in the field.
\end{abstract}

(C) 2014 Elsevier Ltd. All rights reserved.

\section{Introduction}

This paper celebrates Crossley's early disclosure of a new path which guided, since the Sixties and thenceforth, most researchers to understand some fundamental and intrinsic properties of kinematic chains.

So many researchers were inspired by Crossley's new way, founded on Graph Theory, and so several, fresh new, algorithms, most of which are still adopted extensively nowadays both in research and industrial applications, bloomed in literature.

The Graph Theory'smain contribution to Mechanism Science is known to be on Number and Type Synthesis. This topic will be presented in detail by Yan et al. in another paper from the present special issue.

However, the introduction of Graph Theory in the study of mechanisms (including both ordinary and epicyclic gear trains) gave a significant impulse to the development of computer based tools for the automatic generation of kinematic, static force and dynamic equations of any mechanism, on the basis of its corresponding graph, and for the computation of power flow and the mechanical efficiency analysis of gear trains. Indeed, the present paper deals with these collateral effects of the application of Graph Theory to Mechanism Science.

The above mentioned algorithms have a common root with Number and Type Synthesis that consists in the significant opportunities of using concepts, theorems, properties and corollaries borrowed from the Graph Theory.

\footnotetext{
* Corresponding author.

E-mail address: pennestr@uniroma2.it (E. Pennestrì).
} 


\section{Dobrjanskyj's and Freudenstein's early contributions}

The appearance of Graph Theory in Mechanism Science has been quite formally associated (see for example Kota's contribution in a tribute volume published in 1993 [1]) to the innovative work of Dobrjanskyj and Freudenstein [2-4]. However, they make a reference to an earlier systematic classification of the property of graphs, for the first time introduced, in the context of Mechanism Science, by Crossley [5] in 1964. Such paper is very central for the sake of the present review and, so it will be described in Section 3.2.

There are also previous works which gave clear hints about the opportunities offered by the Graph Theory to many fields. However, only a few make reference to mechanics. For example, in 1959 Auslander and Trent began their paper [6] explicitly referring to the study of electrical networks, kinematic mechanisms, traffic control and lumped physical systems. This paper offered a variety of definitions and theorems but no example was applied to the study of mechanisms, although Trent had worked on isomorphisms between graphs and lumped physical systems since 1955 [7].

In 1966 a pioneering work [4] enumerated and specified groups of basic kinematic chains given the degree of freedom $F$, the number of links $l$, of joints $j$ and of $k$-nary links $j_{k}$ and one year later, in 1967, the landmark paper titled Some Applications of Graph Theory to the Structural Analysis of Mechanismsappeared [3]. In this article Dobrjanskyj and Freudenstein applied many concepts of the Graph Theory to suggest a new philosophy of knowledge progress based on the extensive use of the computer, which, at that time, was not so obvious at all. The structure of a kinematic chain was defined by its kinematic graph in which the links are represented by vertices, the joints by edges, and the edge connection between vertices corresponds to the joint coupling of links[4]. Three particular targets were pointed out, together with three original and fundamental methods specifically developed for these purposes:

- determination of structural identity of kinematic chains by means of the identification of isomorphism

- automatic representation of kinematic chains and mechanisms, starting from the incidence matrix

- systematic enumeration of general, single-loop constrained spatial mechanisms.

Starting from the Sixties, the use of the Graph Theory broke out in Mechanism Science and in the Eighties and Nineties became one of the most popular topics in the community, until nowadays, when theGraph Theory has become a classic issue.

\section{Crossley's contribution to the development of graph based algorithms for the analysis of mechanisms and gear trains}

Among Crossley's distinguished contributions [5,8-20] two of the earliest were dedicated to Number and Type Synthesis[9,5]. In the discussions related to the first one [9] a reference to the Graph Theory was indirectly proposed, while in the second one [5] a detailed and rigorous description of the use of the Graph Theory in Mechanism Science was provided, disclosing a very appealing and powerful method that was on the point to become the standard way of describing a mechanism topology.

\subsection{A Contribution to Gruebler's Theory in the Number Synthesis of Plane Mechanisms}

On November 30th, 1961 a new manuscript titled A Contribution to Gruebler's Theory in the Number Synthesis of Plane Mechanisms was received at ASME Headquarters. This work was presented next year, 1962, in New York, on a day between 25th and 30th of November, at the Winter Annual Meeting, and referenced as Paper No. 62-WA-119 ${ }^{1}$. Finally, the paper appeared in the ASME J. of Engineering for Industry in 1964 [9].

The paper, well known within the Mechanism Science Community, described an algorithm which can be employed to solve Gruebler's equation for kinematic chains and presented several new and interesting results. Particularly valuable are Tables 1 and 2 , which show the varieties of the kinematic chains with 1 and 2 degrees of freedom (DoF). Several examples of plane kinematic chains were presented and some critical aspects concerning their mobility were introduced. As usual, a discussion section was appended at the end of the paper, to whom Hain, Jensen and de Jonge contributed, raising many observations and criticisms. The last one focused on the importance of terminology, proposing a distinction between mobility and movability, which moved the attention from kinematic chains to mechanisms. To reply, Crossley's closure ended with the following literally reported statement.

An alternative is to regard them as graphs of undirected planar maps, each with its characteristic incidence of links (edges) and joints (vertices). Here is no dilemma, except in semantics.

Such sentence makes a direct reference to the use of graphs in mechanisms and kinematic chain description and adopts the criterion that joints correspond to vertices, while links correspond to edges.

\subsection{The permutations of kinematic chains of eight members or less from the graph theoretic viewpoint}

In 1964 Crossley wrote what we all know to be a landmark paper titled The Permutations of Kinematic Chains of Eight Members or Less from the Graph Theoretic Viewpoint[5]. This contribution is characterized by solid theoretical bases which much owe to Bill Tutte's foundation works ${ }^{2}$ on graph theory [21-23].

\footnotetext{
1 Such paper no. was used by Crossley himself to reference his previous work in his following paper [5].

2 William Thomas Tutte, Newmarket, Suffolk, England, 1917 - Waterloo, ON, Canada, 2002.
} 
Table 1

List of issues and open problems.

\begin{tabular}{lll}
\hline Topic & Achieved level & Completeness \\
\hline KA2D & Mature & Practically complete \\
DA & Very promising & Open to improvements \\
ASk & Very promising & Lack of procedures for mechanism representation \\
PTGk & Mature & Quite complete \\
PTGeff & Mature & Quite complete \\
T2F & Promising but uncertain & Completely open \\
\hline
\end{tabular}

The following sub-paragraphs describe analytically some fundamental contributions proposed by Crossley in this paper [5]. They allow us to get an idea of the great impact they would have later in the future. Their contents will refer to the same paper [5] unless specified differently.

\subsubsection{Adjacency and incidence matrix, and other data structures}

Terminology and general data structures used in the Graph Theory are described rigorously. The definition of graph $G$ explains how this object has an abstract nature, which is different from the physical system it represents and, also, from any of its representations on a surface. The reader has all the elements necessary to understand the basic rules through many definitions, and so he or she learns that:

- adjacent vertices $v_{j}$ and $v_{k}$ are joined by an edge $e_{j k}$;

- vertices $v_{j}$ and $v_{k}$ are incident to the edge $e_{j k}$;

- an edge is said a simple edge if and only if it consists of one single edge which joins two adjacent vertices;

- a complete graph has an edge for every possible pairing of its set of vertices;

- two graphs $G$ and $G^{\prime}$ are isomorphic when there exists a one to one correspondence between their vertex set $V$ and $V^{\prime}$, such that for every vertex in $G$ there can be found a corresponding vertex in $G^{\prime}$, when also corresponding vertices are joined by edges in $G^{\prime}$ when and only when they are so joined in $G$;

- a path is a sequence of edges $P=\left(\ldots, e_{01}, e_{12}, e_{23}, \ldots\right)$ such that each pair of successive edges has a common endpoint;

- the length of a path is the number of edges contained in it;

- the valency of a vertex is the number of edges incident to it;

- the incidence number $v$ is the number of time a certain edge (or face) is incident to a chosen vertex (or edge); it has been used essentially to represent vertex-edge incidence and so $\nu\left(v_{j}, e_{k}\right)=0$ or 1 accordingly as $v_{j}$ is not incident, or incident, with edge $e_{k}$;

- in an isokinetic chain of $F$ DoF there exists no subassembly of links which, when considered alone, would form a kinematic chain with less that of $F$ DoF.

The latter restriction, which has been mentioned also by Buchsbaum and Freudenstein [24] when defining the main features of graphs representing geared kinematic chains, would be used many more times in the future to study the kinematic chain mobility.

After these definitions, the preferred data structure to represent kinematic chains and mechanisms was, quite naturally, the edgevertex incidence matrix $M_{e v}$, whose entry $M_{e v}(k, j)$ is coincident with the incidence number $\nu\left(e_{k}, v_{j}\right)$. As an alternative, Crossley suggests the adjacency (square) matrix $M_{v v}$, which can be arranged in such a way that the generic element $M_{v v}(i, k)$ carries a 1 whenever $v_{i}$ and $v_{k}$ are joined by an edge.

Some other definitions have been also introduced and the following are intriguing because they have been seldom used in mechanism science:

- a loop is a special edge whose endpoints are identical $e_{k k}$;

- multiple edges join two adjacent vertices;

Table 2

List of contributions on the analysis of planetary gear trains based on the correspondence graph-mechanism.

\begin{tabular}{|c|c|c|c|c|}
\hline \multirow[t]{2}{*}{ Topic } & \multicolumn{4}{|l|}{ Contributions } \\
\hline & 1971-1981 & 1982-1991 & $1992-2002$ & 2003-2013 \\
\hline K.A.PSGT ${ }^{\mathrm{a}}$ & {$[88,106,115]$} & [116-118] & $\begin{array}{l}{[119-122]} \\
{[126-129]}\end{array}$ & $\begin{array}{l}{[95,123-125]} \\
{[130-133]}\end{array}$ \\
\hline K.A. BGT ${ }^{\mathrm{b}}$ & {$[106,134,135]$} & {$[136,137]$} & {$[138,139,29,140]$} & {$[141-146,107]$} \\
\hline P.F. PSGT ${ }^{\mathrm{c}}$ & {$[147,148]$} & & {$[121,108,149,150,31,151]$} & {$[152,153,130,154]$} \\
\hline P.F. BGT ${ }^{d}$ & & & [155] & {$[156,142,157,107]$} \\
\hline M.E. ${ }^{\mathrm{e}}$ & & & [111] & $\begin{array}{l}{[158,159,156,152,160,161]} \\
{[162-164,157,110,133]}\end{array}$ \\
\hline
\end{tabular}

a K.A. PSGT: kinematic analysis of planetary spur gear trains.

b K.A. BGT: kinematic analysis of bevel gear trains.

c P.F. PSGT: power flow analysis of planetary spur gear trains.

d P.F. BGT: power flow analysis of bevel gear trains.

e M.E.: mechanical efficiency analysis. 
- an isolated vertex is disjointed from all the other vertices;

- a graph is separable if its edge set $E$ consists of two disjoint subsets $E_{1}$ and $E_{2}$, with $E=E_{1} \cup E_{2}$, such that there is only one vertex $v$ (called cut vertex) incident to both an edge in $E_{1}$ and in $E_{2}$;

- a bridge is an edge $e_{a b}$ whose removal decomposes the graph into two disjointed subgraphs $G_{1}$ and $G_{2}$ (each at least containing one vertex) being $a \in G_{1}$ and $b \in G_{2}$;

- non-zero elements in the principal diagonal of the adjacency matrix could be used to characterize corresponding special vertices, as they were incident to a loop;

- elements greater than one in the adjacency matrix could represent the presence of multiple edges.

\subsubsection{Structure-to-function mapping}

In 1969 Buchsbaum and Freudenstein [24] developed methods for the classification and enumeration of mechanisms, particularly suitable for gear transmissions and differential drives. In the paper, graphs were extensively used to represent the topological characteristics of the mechanisms and the separation of the kinematic structure from functional considerations was a fundamental idea to provide a designer with a new tool during the conceptual stage of mechanical design. Using graphs and combinatorial analysis the authors managed to enumerate systematically many classes of mechanisms.

In 1979 Freudenstein and Maki [25] suggested a new method for the classification of mechanisms and for the representation of the corresponding kinematic structures and graphs. By using the concept of separation of structure from function shaft couplings and other mechanism were created. Furthermore, the adopted method of creative analysis was suggested for patent interpretation and classification.

The success obtained by Buchsbaum, Freudenstein, Maki and, later, by Mayourian [26], contributed to convey the idea that it is always convenient to separate the concept of structure from function. However, Crossley's very first lines of his paper [5] pointed out a rather different direction. He writes: the desired motion is analysed so as to find the simplest type (or types) of mechanisms that are capable of producing it, or something approximating to it. For example, if it is intended that a moving point shall follow or produce a given curve, the algebraic order of the curve is first noted, and from this the minimum number of links of which the linkage is composed is known immediately. Hence, it is clear that Crossley had in mind a strict relation between motion and structure which, to the best of authors' knowledge, has not been investigated much until nowadays, maybe because the opposite philosophy based on structure and function separation had overwhelmed it by quality and quantity of results.

There are few examples, however, which followed Crossley's former trail.

In 1991 [27] the new concept of partial separation between structure and function was suggested and used to develop a new method for the structural synthesis of geared robotic wrists. This concept relies on the assumption that, once the functional requirements and their relationship to kinematic structure have been determined, it is possible to use specific functional requirements before the enumeration step, in order to reduce the dimension of the problem and make any combinatorial algorithm easier to implement. According to the classical creative synthesis procedures, one of which is qualitatively represented in Fig. 1, the separation of structure and function takes advantages from the interdependency of the stages.

On the other hand, the approach based on the partial separation of structure and function may offer some major advantages such as, for example, the pre-labeling stage, during which the dimension of the enumeration problem is significantly reduced by using kinematic or even dynamic properties of the mechanisms that are to be enumerated. This approach is based clearly on topology-tostructure mapping, rather than on their separation. In order to make the concept clearer, let us consider that mechanism Topology deals only with relations between links, that are treated as abstract entities with no dimensions, velocities or masses. Graphs do focus only on relations between abstract objects. In fact, in a mechanism, any link length or mass variation never affects the corresponding graph. This leads to the guideline concept of the present paper that can be summarized in the following statements:

there is a double effect in using graphs or not; working on the graph means, basically, working on topology and producing results with no matter about the other mechanical properties; instead, structure-to-function mapping helps to fill the gap between the immaterial topological characteristics and a mechanism sought mechanical properties.

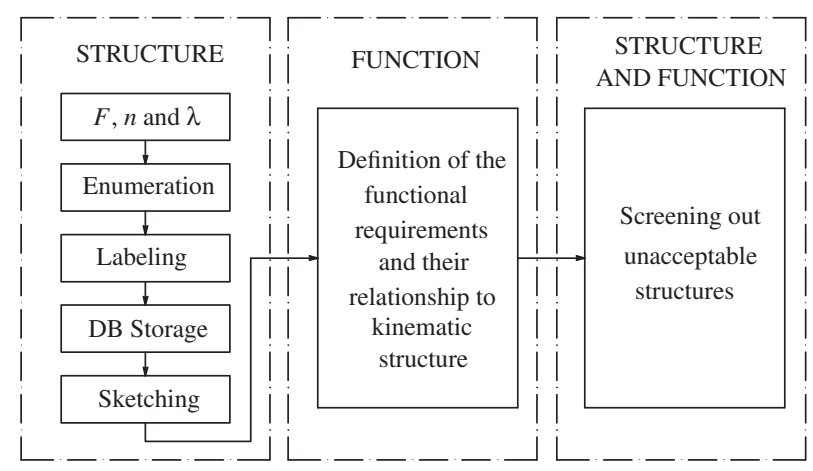

Fig. 1. One example of creative synthesis procedure based on the concept of structure and function separation [25]. 
As mentioned above, such concept was very clear to Crossley since 1964.

By applying the method depicted in Fig. 2 to robotic geared wrists, for example, most of the adjacency matrix entries could be labeled, bypassing the problem of isomorphism, before the enumeration stage. After the enumeration phase, the generated structures were filtered again by making reference to the mechanism transmission line and its feasibility from the kinematic and dynamic viewpoints and, so, a complete census could be sketched $[27,28]$.

In 1995 [29] automatic drive transmissions were analyzed by considering topology together with kinematics and power flows. Hence, the kinematic structure, the gear ratios, the clutching sequence and the circulating power flows could be simultaneously optimized. Starting from Tsai's inventory of planetary gear trains for automatic transmission [30], the dimensional synthesis was applied to multiple-speed automatic transmissions for prescribed velocity ratios and minimum circulating power. For the given displacement graphs, the method exploited all the feasible clutching sequences and, then, computed the gear ratios to achieve the prescribed overall input-output velocity ratios. In order to detect the gear drive with the lowest circulating power, a power-flow analysis was completed for each component of the solution set.

In 1998 Hsieh and Tsai [31] described a procedure for the selection of a most efficient clutching sequence associated with an epicyclic-type automatic transmission mechanism, taking into account the analysis of torque distribution on the links and the power loss associated with various clutching sequences.

Function-related conceptual and creative designs have been attracting constantly researchers from all over the world and are still discussed [32-42].

\subsubsection{Planar vs. plane kinematic chains}

The terms plane and planar are clearly referred to two distinct concepts: the former has to be used for the sake of the geometrical property of a real kinematic chain, while the latter describes the topological properties of a graph and its possibility of being represented on a surface with no line crossing.

Unfortunately, in Mechanism Science, such words have been treated as synonymous by several researchers. As a matter of fact, although a brief note [43] published in 2000 made an attempt to clarify such a distinction, the two terms plane and planar are still used nowadays with the mentioned lack of distinction.

It is therefore quite amazing that the first line of the abstract of Ref. [5] started with mentioning ... in any plane kinematic chain with one degree ... and not ... in any planar kinematic chain with one degree .... As (maybe) known and as Crossley points out on page 473 a graph is called planar if it can be drawn in a plane in such a way that whenever edges meets or cross, there is a vertex and so at least one representation does exist where no crossing edges appear. The introduction, or requirement, of this feature in a graph would be used later to obtain algorithms of automatic sketching of kinematic chains and mechanisms [44-68].

\subsubsection{Independent loop set}

The concept of path has been introduced by Crossley [5] within the context of mechanisms as the sequence of edges such that each pair of successive edges has a common endpoint. From this one, some more fundamental concepts have been presented, such as:

- connected graph, in which exists a path from every vertex to any other;

- circuit, which consists in a cyclic path from any vertex point $a$ through another point $b$ returning to $a$, in which no vertex is passed more than once;

- circuit or path length, which is the number of edges contained in the path;

- the rank $C$ of a connected graph $G$, which is defined by the formula

$$
V-E+C=1
$$

where $V$ and $E$ represent, respectively, the number of vertices and edges in $G$.

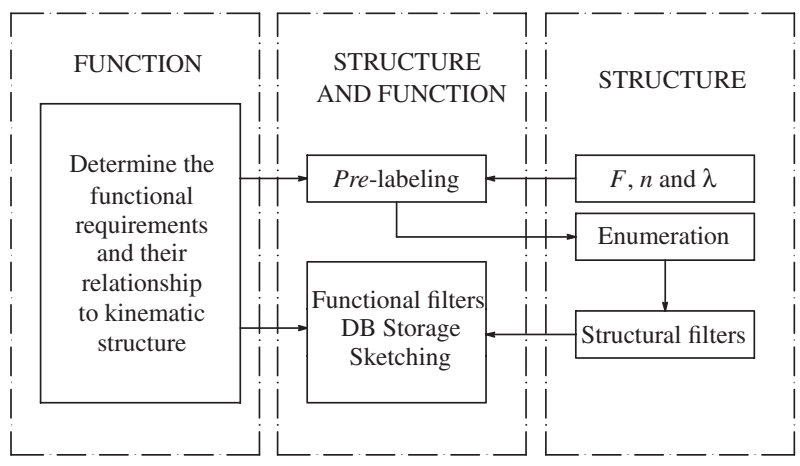

Fig. 2. One example of creative synthesis procedure based on the partial separation of structure and function [27]. 
As pointed out by Crossley, from Paul's previous paper [69], the number of independent circuits $L_{\text {ind }}$, coincident with $C$, is consistent with Euler's polyhedron formula

$$
L_{\text {ind }}=j-\ell+1
$$

where $j$ and $\ell$ are, respectively, the number of kinematic pairs (edges) and links (vertices).

In 1968 Manolescu [70] adopted this formula for mobility and kinematic analysis and so, later on, Topology, Mobility and Connectivity of a kinematic chain were also investigated by using the concept of a set of fundamental circuits (see for example Ref. [71-87]).

The set of independent loops has been used also for many other purposes, as better explained in the following two sections.

\subsubsection{Interchange graph}

Another fundamental tool which appeared in Ref. [5] was the concept of interchange graph, better known as line graph. If planarity gives guarantees that a kinematic chain can be drawn on a surface without edge crossing, the interchange graph is actually the means by which a kinematic chain can be represented for a given corresponding graph $G$. Therefore, this concept has been related mainly to the problem of automatic sketching of kinematic chains and mechanisms.

The interchange graph $L(G)$, or line graph, of graph $G$ is obtained by assigning to $L(G)$ a vertex-vertex adjacency matrix which is coincident to the edge-edge incidence matrix of $G$.

One of the most popular ways to draw a kinematic chain consists in the choice of representing binary links by means of lines, and multiple links by means of polygon having as many edges as the order of multiplicity (and so, for example, a ternary link is represented by a triangle). Once again, Crossley anticipated the idea that the drawing of the line graph $L(G)$ of a kinematic chain represented by graph $G$ is coincident with the polygonal representation of the kinematic chain itself. He had also very clear in mind the difference between graph $G$, as an abstract object, and one of its infinite representations on a surface. For example, consider graph $G$ represented in Fig. 3a). Now, the line graph $L(G)$ of $G$ is obtained by introducing in $L(G)$ one vertex for each edge of $G$ and one edge (in $L(G)$ ) for each vertex of $G$. One possible representation of $L(G)$ is reported in Fig. $3 b)$. Crossley also anticipated, with decades in advance, some fundamental issues in kinematic chain drawing, namely, the problem of unwanted edges. As a matter of fact, he warned all of us about the fact that links with multiplicity $\mu>3$ correspond to a complete graph $K_{\mu}$ and so in the actual representation of $L(G)$ more edges appear than those required to draw the convex hull of a multiple link. For example, graph $G$ reported in Fig. 3 has three multiple links, two of which have multiplicity orders equal to 4 and 5, respectively. As shown in the figure, once $L(G)$ is drawn, as in Fig. $3 b$ ), two and five crossing edges appear within the convex regions which represent, respectively, the quaternary and the quinary links. Unfortunately, the identification of unwanted crossing lines is complicated, since the crossing edges cannot be absolutely defined, rather they are being dependent on the circuits' distribution on the representation surface. For example, consider the different representation of $G$ reported in Fig. $4 a$ ). It is essential to understand that although they look different, the drawings displayed in Figs. $3 a$ ) and $4 a$ ) do refer to exactly the same graph $G$ and they differ only because the circuits have been laid out differently. According to the reported example, the quaternary link remains unchanged, while some edges that were unwanted (dash-dot lines) in the former representation become external, and so, necessary to represent the 5-edge polygon, in the second lay out (see Fig. $4 b$ )).

\subsubsection{The identification of the transfer vertex in epicyclic gear trains}

Although the Graph Theory has been used mainly for the structural synthesis, a certain success has been achieved in using it for the kinematic and static force analyses for Epicycilc Gear Trains (EGTs). This achievement was possible mainly because the new data structures could be adopted to generate automatically the equations.

Since 1970 [24]the Graph Theory has been used successfully for the structural synthesis of EGTs and one year later Freudenstein [88] and, then, Yang [89] suggested a systematic procedure for kinematic analysis, force analysis and power-flow determination. The method was based on some characteristic properties of the labeled graph corresponding to any EGT, namely, the existence of a characteristic spanning tree which simplifies the detection of a particular set of fundamental circuits.

A connected undirected graph with no circuits is said to be a tree. A spanning tree of graph $G$ is a subgraph of $G$ which has all the vertices of $G$ and a maximal set of edges of $G$ that contains no cycle.
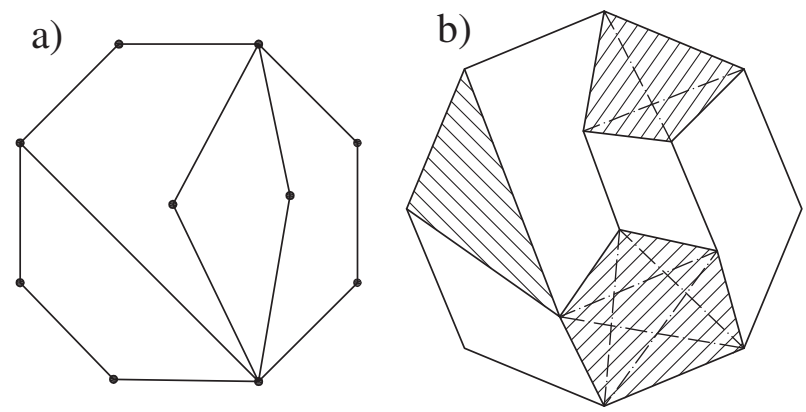

Fig. 3. The line graph $L(G) b$ ) and its corresponding graph $G a$ ). 

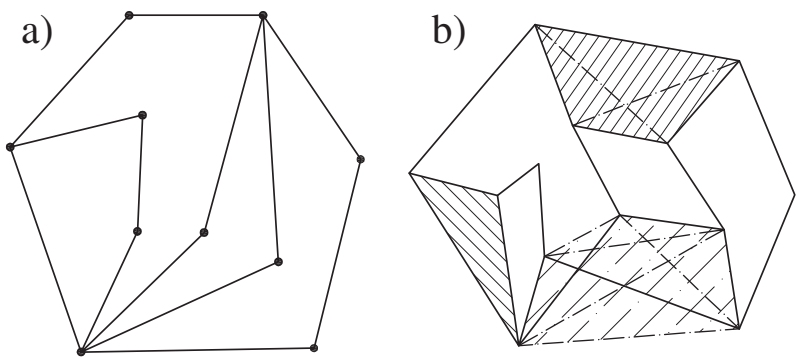

Fig. 4. Another representation of $G a$ ) and $L(G) b$ ) of the graph depicted in Fig. 3a).

After removal of all the geared edges from the graph of an EGT, a particular spanning tree is obtained, while all the fundamental circuits can be achieved by repositioning back in the spanning tree one geared edge at the time. The fundamental circuits, so obtained, can be analyzed in order to identify the gear carriers embedded in the structure, which makes it possible to apply Willis' equations $L_{i n d}$ times and, so, to generate the full set of kinematic equations. The identification of the gear carriers in the structure can be also used to write the static force equations. Finally, power flows and efficiency can be also calculated.

Starting from Crossley's definitions [5], Freudenstein [88], as above mentioned, suggested a way to solve the problem of the automatic generation of the equations for kinematic, static forces and power flow analysis. However, the main obstacle to the development of a computer code was how to identify automatically the set of the fundamental circuits and the corresponding transfer vertices which, as it will be explained later, represent the gear carriers.

In 1989 [90] a new method based on a particular group of data structures was suggested. According to the method, the fundamental circuits and the transfer vertices could be identified by means of a graph traversal and the selective visit of the nodes belonging to the spanning tree. The results of such traversal could be stored in a two-field-record list which was visited by using pointer variables. The obtained lists could be used also to generate the equations.

Some years later, in 1995, Tsai [91] suggested a completely different approach based on the following concepts:

- canonical graph, which is obtained by reconfiguring the revolute joints among the coaxial links without affecting the functionality of the mechanism;

- directed graph, which is a new graph obtained by an undirected graph by assigning a direction to the edges;

- edge set matrix, which is a $3 \times E($ or $3 \times j)$ matrix whose columns represent edges: an element of the first and second rows represents the tail and head edges and the third element is the edge label;

- circuit matrix, whose generic element $C(i, j)$ is 1 (true) if edge $i$ forms part of the boundary for circuit $j, 0$ otherwise;

- path matrix, whose element $T(i, j)$ is equal to: +1 if edge $i$ is on the path from the root (of the tree) to vertex $j$ and the edge is directed toward the root; -1 if edge $i$ is on the path from the root to vertex $j$ and the edge is directed away from the root; 0 otherwise.

These data structures have been reported herein because they have not been fully investigated and so they could suggest to the readers new ideas for research work. Indeed, by manipulating these matrices Tsai was able to build a brilliant procedure for the automatic detection of the transfer vertex.

\subsection{The relevance of Crossley's early contributions as triggering events}

In the early Sixties, the Graph Theory was not an established subject as we know it today and so, all the concepts described in the present paragraphs have to be considered in the context of an early and creative season for mechanism topology. Number and Type Synthesis have to be considered as the natural development of the introduction of the Graph Theory in Mechanism Science. However, apart from these important topics, there were others that were worth to be studied.

The following is a list of topics and problems in which the Graph Theory can be conveniently involved.

KA2D kinematic analysis of plane mechanisms: this topic is nowadays well known also at high educational level; some improvements are still possible but the fundamentals have been established so far;

DA dynamic analysis and simulation of mechanisms: there are some algorithms taken both from graph and network theories which were helpful to identify the interactions between each body and the other ones from the system, including the body interactions with the inertial reference frame; such procedures could be developed and expanded to other methods for the dynamic analysis of multi-body systems;

ASk automatic sketching of planar kinematic chains and mechanisms: there are several contributions dedicated to the kinematic chains, most of which provides, within a reasonable CPU time, nice and accurate planar drawings; however, there is a real want of algorithms for the automatic sketching of mechanisms;

PTGk kinematic, static force, power-flow analysis of epicyclic gear trains: there are many algorithms available which are able to handle symbolic and numeric calculations with certain reliability; those which make use of the Graph Theory are more general;

PTGeff efficiency and optimization of epicyclic gear trains: efficiency and optimization of planetary gear trains have been investigated extensively; the methods which make use of Graph Theory show their attitude to be employed in the general cases; 
T2F topology-to-function mapping: this topic is quite new; actually there are no systematic methods available for predicting the function for a given topology and vice versa.

Table 1 presents the authors' subjective view of the interest and popularity that the above listed issues have obtained among the Scientific Community, with special regard about quantity and quality of algorithms' availability and study completeness.

\section{Automatic sketching of kinematic chains}

Most valuable results obtained using the Graph Theory could be used for the automatic sketching of kinematic chains and mechanisms. The first useful tool, for example, is the well known planarity test developed by Hopcroft and Tarjan [92].

Starting from the first computer programs [2,3] in 1967, Woo suggested in 1969 [93] an interactive procedure for crossing edge avoidance. In 1984 Mayourian [26] developed a code for the creation of plane mechanisms using the Graph Theory with graphical output consisting of labeled graphs satisfying structural constraints but without considering the problem of edges crossing.

Olson et al. [44] proposed in 1985 a procedure based on heuristic rules adopted to minimize the likelihood of crossing edges. They used Crossley's observation [5] that the sketch of the line graph $L(G)$ of graph $G$ is equivalent to the sketch of the kinematic chain corresponding to graph $G$.

Three years later Chieng and Hoeltzel [45] suggested a combinatorial approach. In 1988 Yang et al. [46,47] introduced new rules for the kinematic sketching of mechanisms although such problems do not admit a unique solution.

In 1989 Yan and Hwang presented a method [48] for the minimization of the number of crossing links and achieving an artistic balance of the sketches. The approach was based on a small number of nice looking hand drawing set of basic contracted kinematic chains, and on some link and joint contractions and expansions.

In 1994 a list of the features a good sketch is expected to show was summarized [49] and, basically, they remained the same until now:

- all the lines must be drawn straight;

- edge crossing must be either minimized or eliminated (if the graph is planar);

- $k$-nary multiple links must be represented by convex polygon with $k$ edges;

- line length ratios must be limited for esthetic reasons;

- the drawing must be displayed in a decent time (i.e. the computational efficiency of the algorithm must be adequate);

- no human action must be required during execution time.

In the same paper [49] the adopted method was based on four main steps and several examples could be provided.

1. Given the edge list of $G$ find any mapping $r(G)$ embedding $G$ with a convex shape;

2. Evaluate the vertex-vertex adjacency matrix of $G$ and of $L(G)$;

3. Delete the extraneous crossing edged in the polygon representing links with multiplicity degree greater than 3 ;

4. Taking into account $r(G)$, draw the links accordingly to $L(G)$;

In 1996 Mauskar and Krishnamurty [52] developed a loop configuration, inter-loop relationship based, approach for automatically sketching every type of kinematic chain regardless of the number of links and degrees of freedom.

The automatic sketching of kinematic chains has been constantly investigated during the following years [50-68] and it is still an open and interesting problem.

\section{Kinematic analysis of plane mechanisms}

The set of fundamental circuits has been used to identify a set of independent equations which can be adopted to solve the position and the first and second order kinematic analyses of plane mechanisms.

The interesting feature consists in the topological approach capability in finding a complete set of independent equations according to a method that can be automated easily and that does not rely on geometrical information. As a matter of fact, finding one graph spanning tree corresponds to identify a set of closed loop equations for the corresponding mechanism. This concept will be here exemplified by means of two examples, one 6-links Stephenson and one 10-links plane mechanisms.
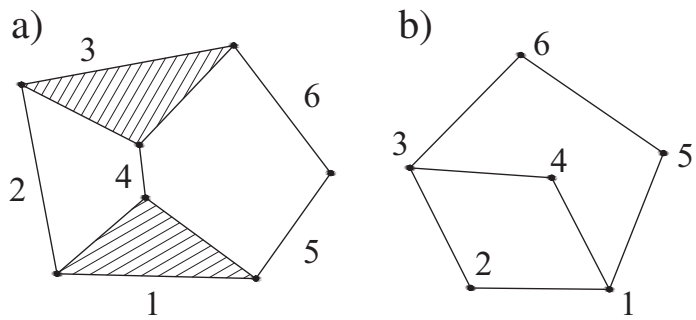

Fig. 5. Stephenson kinematic chain $a$ ) and its corresponding graph $b$ ). 
Stephenson classical kinematic chain is represented in Fig. 5a) while its corresponding graph is reported in Fig. 5b).

One possible mechanism which can be obtained from the Stevenson kinematic chain is reported in Fig. $6 a$ ) where two loops $L_{a}$ and $L_{b}$ can be identified. Introducing oriented distances $\vec{a}_{i}$ and $\vec{b}_{i}$ from and to the pin joint centers, as shown in Fig. $6 b$ ), the two equations

$$
\begin{aligned}
& \vec{a}_{1}+\vec{a}_{4}+\vec{a}_{3}+\vec{a}_{2}=0 \\
& \vec{b}_{1}+\vec{b}_{5}+\vec{b}_{6}+\vec{b}_{3}+\vec{b}_{4}=0
\end{aligned}
$$

can be easily written, respectively, for loops $L_{a}$ and $L_{b}$.

The task of finding the fundamental circuits may seem trivial for the most simple mechanisms because the solution is obtained by a simple visual inspection. However, when the process automation is required, for example, when general purpose software packages acquire topological and geometrical information from user's friendly interactive procedures, the seeking of the fundamental circuit requires specific algorithms from the Graph Theory. For the mechanism reported in Fig. 6a) the graph represented in Fig. 5b) can be manipulated to obtain one spanning tree, as the one represented in Fig. $7 a$ ), whose edges are called branches. As described above, when one of the removed arcs, called chords, is reintegrated back in the graph in its original position, the spanning tree becomes a graph which has only one loop and this loop is exactly the one which is completed by the removed arc reintegration. This property is depicted in Fig. $7 b$ ).

Another kinematic chain is reported in Fig. 8a) together with its corresponding graph (see Fig. 8b)). Fig. 9a) shows one possible mechanism obtained from this kinematic chain. The loops represented in Fig. $9 b$ ) can be obtained by using the spanning tree reported in Fig. 10a) and the following set of equations

$$
\begin{aligned}
& \vec{a}_{1}+\vec{a}_{10}+\vec{a}_{9}+\vec{a}_{7}=0 \\
& \vec{b}_{7}+\vec{b}_{6}+\vec{b}_{5}+\vec{b}_{4}=0 \\
& \vec{c}_{1}+\vec{c}_{7}+\vec{c}_{4}+\vec{c}_{8}=0 \\
& \vec{d}_{1}+\vec{d}_{7}+\vec{d}_{4}+\vec{d}_{3}+\vec{d}_{2}=0
\end{aligned}
$$

can be written.

Fig. 10b) shows how the reintegration of chords 3-4 into the spanning tree (reported in Fig. 10a)) allows us to identify the unique loop $L_{d}$. The same applies to the other circuits $L_{a}, L_{b}$, and $L_{c}$ when chords 7-9, 4-5 and 4-8, respectively, are returned back to their original positions.

As clearly pointed out by Paul [69] in 1960, the $L_{\text {ind }}$ closed-loop equations correspond, in the plane, to $2 L_{\text {ind }}$ Scalar equations which can systematically formulate the governing kinematic equations of the mechanism.

In 1962 Sandor [94] presented a method based on the set of independent closed-loop equations for obtaining the first, second and higher order loop equations for any plane mechanism composed of one or more closed loop of successive bar-slider members.

Finally, an interesting and general interpretation on the use of graphs in kinematic and dynamic analysis was offered by Shai and Pennock [95]. They introduced new graph representations of mechanical systems to provide more insights into the duality of statics of structures and the kinematics of mechanisms. Thanks to this duality it was possible to detect a parallelism between the kinematics of a planetary of a gear train and the statics of a determinate beam.

a)

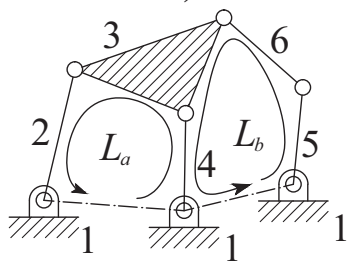

b)

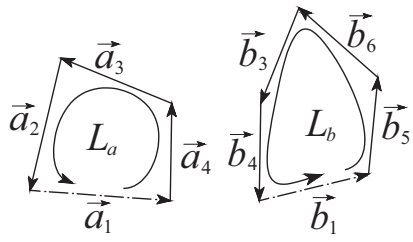

Fig. 6. One possible mechanism $a$ ) obtained from the Stephenson kinematic chain and its corresponding loops $L_{a}$ and $L_{b} b$ ). 
a)

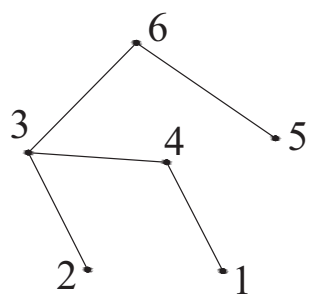

b)

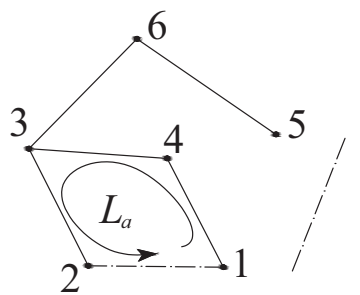

Fig. 7. One spanning tree $a$ ) of the graph reported in Fig. 5b) and its removed chords (dash dotted lines) $b$ ).

\section{Dynamic analysis and simulation of multi-body systems}

For the last decades many methods for the automatic formulation of the equations needed to simulate the dynamic behavior of a multi-body system have been proposed. The Graph Theory has been used also for the dynamic analysis and simulation of multi-body systems. Among the first proposals Wittenburg, since 1977 [96], built a method, based on the Graph Theory, for the formulation of the governing equations of motion, for both open and closed-loop kinematic chains, where rigid bodies were represented as nodes, while the edges corresponded to the connections (or hinges) between bodies. According to this formulation, the graph is used to obtain the necessary topological relationships between the bodies, while classical dynamics, such as Newton's Laws and D'Alembert's Principle, provide the remaining equations of motions.

It should be acknowledged that the use of graphs to represent system topology during the generation of the governing equations of motion in multi-body dynamic systems has been exploited by Andrews and his coworkers [97-100], Sheth and Uicker [101] and by Wittenburg [102,103]. In Wittenburg's graph rigid bodies represent not only graph vertices and the edges of the kinematic pairs, but also spring-damper elements. However, his graph representation has some shortcomings for generating loop closure equations.

Later, in 1996, McPhee et al. noticed [103] that this method was not easy to be fully automated and therefore pointed out a different approach called vector-network technique, according to which the equations governing the motion of a large class of mechanisms, namely, the tree-structured systems, could be obtained by writing the cutset equations for an augmented form of Wittenburg's graph that contains mass and force elements. In order to explain better this concept, some more properties and definitions will be herein added, to those already introduced in Sections3.2.6 and 5:

- the fundamental circuits form a basis for the circuit space;

- a cut-set of a connected graph is a set of edges whose removal would disconnect the graph;

- any branch edge $e$ of a spanning tree $T$ defines a partition of the graph vertices $V$ in two distinct sets, say, $V_{1}$ and $V_{2}$;

- a fundamental cut-set is the cut-set which contains only the branch edge $e$ and some chord edges chosen in such a way that the cutset yields the same partition in $V_{1}$ and $V_{2}$.

As suggested in the abovementioned paper [103], when the system consists in closed loop mechanisms, the concept of fundamental circuits is helpful in finding a set of closed-loop equations which constrain the system dynamics, while the concept of fundamental cutset can be applied to the vector-network diagram, to represent, essentially, the moment or force that would be obtained from a freebody diagram. The fundamental cut-set is needed, in particular, in order to select, from the whole set of inertial and external forces and torques, those which affect each single body.

\section{Analysis of planetary gear trains}

Gear trains can be considered representative of a class of linear mechanical systems. This fully justifies the widespread development of analysis methodologies of graphs for their modeling. The possibility to represent in an abstract manner the kinematic

a)

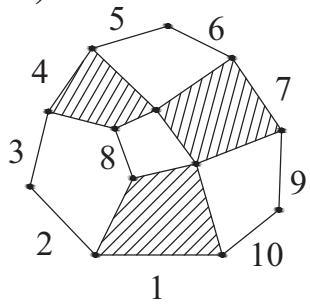

b)

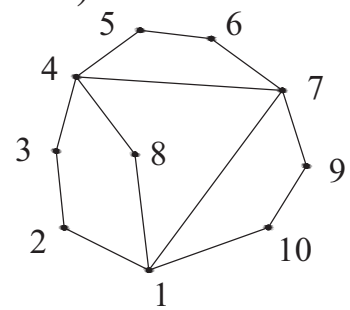

Fig. 8. A 10-link kinematic chain $a$ ) and its corresponding graph $b$ ). 
a)

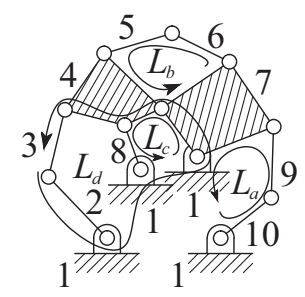

b)

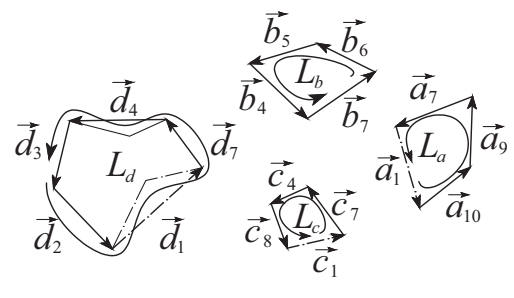

Fig. 9. One possible mechanism $a$ ) obtained from the kinematic chain represented in Fig. $8 a$ ) and its corresponding loops $L_{a}, L_{b}, L_{c}$ and $L_{d} b$ ).

structure of a gear train offered new ways of approaching in a very systematic manner different types of analyses on complex planetary gear trains.

A planetary gear train can be considered as a combination of elementary gear trains, this being the kinematic chain composed of two meshing gears and one gear carrier. Once the analysis equations for this chain were established, thanks to linearity, the full set of equations for the complex gear train could be generated.

To the best of our knowledge, the first comprehensive analysis study which considers the gear train as a linear network dates back to 1969 and is due to Polder [104] who in his dissertation states:

Epicyclic gear trains and nodes have in common that they interconnect three power transmitting shaft ends, thus constituting three-poles in a variator network. No other entities than three-poles are required to perform and schematically represent the interconnections of rotating shafts. If more than three shafts meet, they can be described by a sequence of three poles.

This description somewhat anticipates graph representation of a geared chain and the concept of fundamental circuit used by Freudenstein and Buchsbaum [24] for the purpose of enumeration. In this paragraph, the main kinematic structure properties of a geared chain will be listed too.

The topology of a gear train is meaningfully identified by means of a labeled graph where links are represented by vertices and kinematic pairs by edges, respectively. For geared chains $L_{\text {ind }}$ coincides with the number $j_{G}$ of gear pairs. As a consequence, the Gruebler's equation for the computation of the degrees-of-freedom $F$, taking into account Eq. (2), simplifies into

$$
F=j_{R}-j_{G},
$$

where $j_{R}$ and $j_{G}$ are the number of revolute and geared pairs, respectively.

Edges are labeled according to the type of kinematic pair, i.e. $R$ : revolute pairs and $G$ : gear pair. A letter within parentheses specifies the axis level of the revolute pair.

This approach allows the location of thefundamental circuits (Fig. 11) in the planetary gear train graph. The fundamental circuit identifies the simplest gear unit, that is two meshing gears connected through a gear carrier.

By identifying the fundamental circuits one recognizes the elementary gear units within the gear train and the system of analysis is systematically generated.

The introduction of the fundamental circuit concept for kinematic analysis of gear trains is due to Freudenstein [88] who, making a direct reference to Crossley [5], states

...it is useful to define the graph of a mechanism as a linear graph in which the links correspond to vertices, the kinematic pairs to edges, and the pair-connection between links corresponds to the edge-connection between vertices.

Since then many investigations adopted the correspondence graph-mechanisms suggested by Crossley as a tool to obtain the analysis equations. A review on the evolution of graph based representation of gear trains has been presented by Wojnarowski et al. [105].

a)

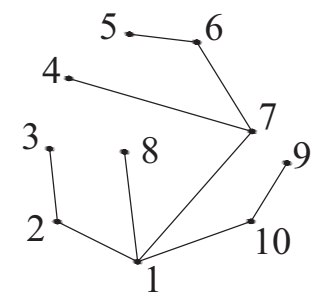

b)

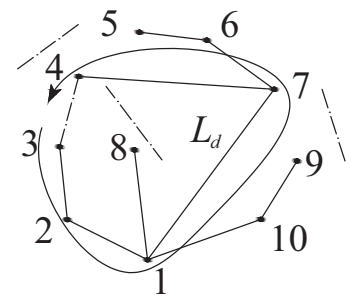

Fig. 10. One spanning tree $a$ ) of the graph reported in Fig. 8b) and its removed chords (dash dotted lines) $b$ ). 

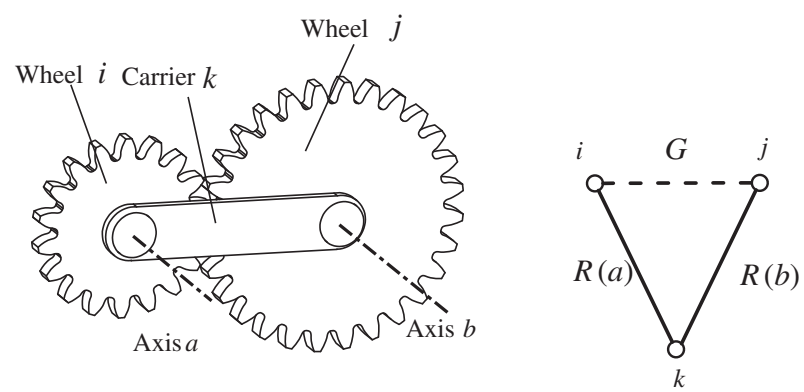

Fig. 11. Labeled graph representation of a fundamental circuit. $G$ : gear pair; $R(a), R(b)$ : revolute pairs with different axes; $i$ and $j$, gear wheels; $k$ gear carrier (transfer vertex).

For the purpose of kinematic analysis of spur planetary gear trains, the main equation follows from the so called Willis' equation [106]

$$
\omega_{i}-R_{m} \omega_{j}+\left(R_{m}-1\right) \omega_{k}=0
$$

where $\omega_{i}, \omega_{j}$ and $\omega_{k}$ denote the absolute angular velocities of the wheels $i, j$ and gear carrier, respectively, and

$$
R_{m}= \pm \frac{z_{j m}}{z_{\text {im }}}= \pm \frac{\text { Number of teeth of wheel } i}{\text { Number of teeth of wheel } j}
$$

is the gear ratio.

An extension of Willis' equation to the case of bevel gears is introduced in [107].

For the purpose of power flow analysis, we can distinguish two types of relations:

- a power balance at the $n$th node;

$$
\sum_{u \in U} P_{n, u}=0
$$

where $U$ is the set of all independent loops that contain such node;

- a power balance within the links of the $m$ th elementary gear train

$$
P_{i, m}+P_{j, m}+P_{k, m}=0
$$

where $P_{i, m}, P_{j, m}$ and $P_{k, m}$ are, respectively, the powers through the links $i, j$ and $k$.

These powers are related by means of following [108]

$$
\begin{aligned}
& \frac{P_{j, m}}{P_{i, m}}=-R_{m} \frac{\omega_{j}}{\omega_{i}}, \\
& \frac{P_{k, m}}{P_{j, m}}=\frac{1-R_{m}}{R_{m}} \frac{\omega_{k}}{\omega_{i}} .
\end{aligned}
$$

These analysis equations are applied in a fashion similar to Kirchhoff's laws for electrical circuits [108-110].

Also mechanical efficiency analysis of planetary gear trains benefited of the correspondence graph-mechanisms introduced by Crossley (e.g. [111]).

Table 2 summarizes, grouped by years, graph based contributions to different areas of planetary gear trains. These contributions are based on the graphs derived by those introduced by Crossley. For this reason bond graphs, which are of a different nature, have been omitted. Although great care has been taken to compile the table, the authors cannot ensure exhaustiveness.

Tendon driven mechanisms have been also analyzed with the help of the Graph Theory. The similarity between the analysis methods of gear trains and this class of mechanisms has been recognized by Tsai and Lee [112]. In fact, the belt and the pulleys can be reduced to a higher pair and the link connecting the two pulleys with revolute joints has the same function of the gear carrier. Contributions where the equations of motion of tendon driven mechanisms have been deduced by means of graphs are due to Dunlop and Khajepour [113], Uyguroğlu and Demirel [114]. 


\section{Conclusions}

This paper was written to celebrate Crossley's fundamental intuition of adopting the Graph Theory as a means to model several properties of kinematic chains and mechanisms. For this purpose, this review paper discloses, hopefully, how Crossley's new trial guided many kinematicians not only through the field of Number and Type Synthesis, but also through the study of Kinematic, Static, Dynamic, Power Flow and Efficiency Analysis of Mechanisms and Gear Trains.

Although the main part of this paper contents refers to one of Crossley's earliest paper, back to 1964, the authors, during the development of this review, could not help but notice that many interesting and actual issues are still open, when not unexplored. Hence, they hope that the efforts they made in order to present the Graph Theory related issues in Mechanism Science (other than Number and Type Synthesis) could invite the readers to consider such (still) promising topic and, so, attract their new contributions.

\section{References}

[1] A.G. Erdman, et al., Modern kinematics: developments in the last forty years, Wiley Series in Design Engineering, J. Wiley, New York, 1993. (A WileyInterscience publication).

[2] L. Dobrjanskyj, Application of Graph Theory to the Structural Classification of Mechanisms, (Ph.D. thesis) Columbia University, 1966.

[3] L. Dobrjanskyj, F. Freudenstein, Some applications of Graph Theory to the structural analysis of mechanisms, J. Manuf. Sci. Eng. 89 (1967) 153-158.

[4] F. Freudenstein, L. Dobrjanskyj, On a theory for the type synthesis of mechanisms, in: H. GÃrtler (Ed.), Applied Mechanics, Springer, Berlin Heidelberg, 1966, pp. 420-428.

[5] F.R.E. Crossley, The permutations of kinematic chains of eight members or less from the graph theoretic viewpoint, in: W.A. Shaw (Ed.), Developments in Theoretical and Applied Mechanics, vol. 2, Pergamon Press, Oxford, 1964, pp. 467-486.

[6] L. Auslander, H. Trent, Incidence matrices and linear graphs, Indiana Univ. Math. J. 8 (5) (1959) 827-835.

[7] H.M. Trent, Isomorphism between oriented linear graphs and lumped physical systems, J. Acoust. Soc. Am. 27 (3) (1955) $500-527$.

[8] F.R.E. Crossley, Germen, On the response of the singly free linear oscillator, Int. J. Mech. Sci. 1 (4) (1960) $396-397$.

[9] F.R.E. Crossley, A contribution to Gruebler's theory in the number synthesis of plane mechanisms, Transactions of the ASME, J. Eng. Ind. 86 (1964) 1-8.

[10] F.R.E. Crossley, Corrigendum, J. Mech. 3 (3) (1968) 219.

[11] F.R.E. Crossley, Über die Kräfte in Kombinationen von Gelenk-und Rädertrieben (On the forces which occur in gear-linkage-combination mechanisms). f. Heinrich Lehn: Dr. -Ingenieur Dissertation, Technische Hochschule Aachen, West Germany, July 1967, J. Mech. 5 (2) (1970) 279.

[12] F.R.E. Crossley, Editorial, Mech. Mach. Theory 7 (1) (1972) 1-2.

[13] K.H. Hunt, F.R.E. Crossley, Coefficient of restitution interpreted as damping in vibroimpact, J. Appl. Mech. Trans. ASME Ser. E 42 (2) (1975) 440-445.

[14] R. Azar, F.R.E. Crossley, Experimental investigation of impact phenomenon in spur gear systems, World Congr on the Theory of Mach and Mech, 4th I, 1975, pp. 157-161.

[15] M.A. Veluswami, F.R.E. Crossley, Multiple impacts of a ball between two plates - 1. Some experimental observations, J. Eng. Ind. Trans. ASME Ser. B 97 (3) (1975) 820-827.

[16] M.A. Veluswami, F.R.E. Crossley, G. Horvay, Multiple impacts of a ball between two plates - 2. Mathematical modelling, J. Eng. Ind. Trans. ASME Ser. B 97 (3) (1975) 828-835.

[17] F.R.E. Crossley, F.G. Umholtz, Design for a three-fingered hand, Mech. Mach. Theory 12 (1977) 85-93.

[18] R.C. Azar, F.R.E. Crossley, Digital simulation of impact phenomenon in spur gear systems, J. Eng. Ind. Trans. ASME Ser. B 99 (3) (1977) $792-798$.

[19] F.R.E. Crossley, Nominations for new officers of IFToMM, Mech. Mach. Theory 14 (2) (1979) 155-156.

[20] F.R.E. Crossley, Wood chips - an alternative heating fuel, Mech. Eng. 102 (5) (1980) 36-43.

[21] W.T. Tutte, A census of planar triangulations, Can. J. Math. 14 (1962) 21-38.

[22] W.T. Tutte, A census of Hamiltonian polygons, Can. J. Math. 14 (1962) 402-417.

[23] W.T. Tutte, A census of planar maps, Can. J. Math. 15 (1963) 249-271.

[24] F. Buchsbaum, F. Freudenstein, Synthesis of kinematic structure of geared kinematic chains and other mechanisms, J. Mech. 5 (3) (1970) $357-392$.

[25] F. Freudenstein, E.R. Maki, The creation of mechanisms according to kinematic structure and function, Environ. Plann. B 6 (4) (1979) 375-391.

[26] M. Mayourian, The creation of mechanisms according to the separation of kinematic structure and function and its partial automation, (Ph.D. Thesis) Columbia University, New York, NY, USA, 1985. (aAI8604645).

[27] N.P. Belfiore, L.W. Tsai, A new methodology for structural synthesis of geared robotic wrists, Proc. of the 2nd Nat. Applied Mechanisms and Robotics Conf, 1991.

[28] N.P. Belfiore, Atlas of remote actuated bevel gear wrist mechanisms of up to nine links, Int. J. Robot. Res. 12 (5) (1993) $448-459$.

[29] E. Pennestrì, N.P. Belfiore, Kinematic design and optimization of automatic drive transmissions, J. Appl. Mech. Robot. 2 (4) (1995) 7-14.

[30] L.-W. Tsai, E. Maki, T. Liu, N. Kapil, The categorization of planetary gear trains for automatic transmissions according to kinematic topology, SAE Technical Papers1988. 885062.

[31] H. Hsieh, L.W. Tsai, The selection of a most efficient clutching sequence associated with automatic transmission mechanisms, J. Mech. Des. Trans. ASME 120 (4) (1998) 514-519.

[32] J.K. Shin, S. Krishnamurty, Development of a standard code for colored graphs and its application to kinematic chains, ASME DE, vol. 47,1992 , pp. $247-254$.

[33] V.P. Agrawal, J.N. Yadav, C.R. Pratap, Link-centre and indexes of a kinematic chain, ASME DE, vol. 46, 1992, pp. 121-128.

[34] Y. Meng, C. Wu, Method of conceptual creative design of mechanisms based on combinations of kinematic pairs, Zhongguo Jixie Gongcheng/China Mechanical Engineering 15 (23) (2004) 2088-2091.

[35] H. Ding, Z. Huang, Y. Cao, Topological graphs creation automatically of kinematic chains and atlas database establishment, Jixie Gongcheng Xuebao/Chin. J. Mech. Eng. 42 (4) (2006) 32-36.

[36] H.S. Yan, J.J. Chen, Creative design of a wheel damping mechanism, Mechanism and Machine Theory 20 (6) (1985) 597-600.

[37] C.-C. Hung, H.-S. Yan, G. Pennock, A procedure to count the number of planar mechanisms subject to design constraints from kinematic chains, Mech. Mach. Theory 43 (6) (2008) 676-694.

[38] W.-C. Zhou, H.-H. You, Topological analysis based on the kinematic chain of knitting machine needle guide agency creative design, ICMET 2010, 2010, pp. 364-366.

[39] W.-C. Zhou, B. Wu, The design of ternary joint robot fingers without drive based on the topological graph of kinematic chain, Appl. Mech. Mater. 34-35 (2010) $761-764$.

[40] W.-C. Zhou, F.-X. Zhao, Innovative new-typed mechanism indicating flow by use of topological and structural characteristics and the graph of kinematic chains, ICCDA, vol. 1, 2010, pp. V1177-V1179.

[41] Z. Deng, F. Yang, J. Tao, Adding sub-chain method for structural synthesis of planar closed kinematic chains, Chin. J. Mech. Eng. (Engl. Ed.) 25 (2) (2012) 206-213.

[42] H.-S. Yan, Y.-T. Chiu, An algorithm for the construction of generalized kinematic chains, Mech. Mach. Theory 62 (2013) $75-98$.

[43] N.P. Belfiore, Brief note on the concept of planarity for kinematic chains, Mech. Mach. Theory 35 (12) (2000) 1745-1750.

[44] D. Olson, T. Thompson, D. Riley, A. Erdman, Algorithm for automatic sketching if planar kinematic chains, J. Mech. Transm. Autom. Des. 107 (1) (1985) $106-111$.

[45] W.-H. Chieng, D.A. Hoeltzel, Combinatorial approach to the automatic sketching of planar kinematic chains and epicyclic gear trains, ASME DE, vol. 15-1, 1988, pp. 79-90. 
[46] B. Yang, U. Datta, P. Datseris, Y. Wu, Development of an integrated expert system for kinematic design of mechanisms - theory-2, ASME DE, vol. 15-1, 1988, pp. 91-100.

[47] B. Yang, U. Datta, P. Datseris, Y. Wu, Development of an integrated expert system for kinematic design of mechanisms - applications, ASME DE, vol. 15-1, 1988, pp. 101-109.

[48] H.-S. Yan, Y.-W. Hwang, New algorithm for automatic sketching of kinematic chains, Proc. Int. Computers in Eng. Conf. and Exhibit, 1989, pp. 245-250.

[49] N.P. Belfiore, E. Pennestrì, Automatic sketching of planar kinematic chains, Mech. Mach. Theory 29 (1) (1994) 177-193.

[50] Z. Wang, W. Li, H. Li, R. Wu, Automatic sketching of planar kinematic chains, ASME DE, vol. 71-2, 1994, pp. 433-438.

[51] G. Chatterjee, L.-W. Tsai, Computer-aided sketching of epicyclic-type automatic transmission gear trains, J. Mech. Des. Trans. ASME 118 (3) (1996) $405-411$.

[52] S. Mauskar, S. Krishnamurty, A loop configuration approach to automatic sketching of mechanisms, Mech. Mach. Theory 31 (4) (1996) $423-437$.

[53] S. Lang, H. Kesavan, Graph theoretic modeling and analysis of multibody planar mechanical systems, IEEE Trans. Syst. Man Cybern. Syst. Hum. 31 (2) (2001) $97-111$

[54] Y. Liu, R. Xiao, New approach to automatic sketching of planar kinematic chains based on loop configuration and its computerization, Jixie Gongcheng Xuebao/ Chin. J. Mech. Eng. 38 (6) (2002) 21-25.

[55] R. Xiao, Y. Liu, D. Wang, An improved approach to automatic sketching of mechanism based on loop configuration and its implementation, ASME DE, vol. 5 A, 2002, pp. 655-660.

[56] T. Mruthyunjaya, Kinematic structure of mechanisms revisited, Mech. Mach. Theory 38 (4) (2003) 279-320.

[57] Z. Huang, H. Ding, Y. Cao, The novel characteristic representations of kinematic chains and their applications, ASME DE, vol. 7 A, 2005, pp. 201-210.

[58] I. Popescu, D. Marghitu, The structure of planar mechanisms with dyads, SPIE, vol. 5999, 2005.

[59] G. Chatterjee, Enumeration and Automatic Sketching of Epicyclic-Type Automatic Transmission Gear Trains, Master's Deg. Thesis, M.S. 93-15, Inst. for Systems Research, University of Maryland, 1993.

[60] H. Ding, Z. Huang, A unique representation of the kinematic chain and the atlas database, Mech. Mach. Theory 42 (6) (2007) 637-651.

[61] D. Marghitu, I. Popescu, Structural design of planar mechanisms with dyads, Multibody Syst. Dyn. 19 (4) (2008) 407-425.

[62] S. Nie, H. Liu, A. Qiu, A maximal loop approach to automatic sketching of mechanisms, ASME DE Part A, vol. 9, 2008, pp. 501-509.

[63] S. Nie, H. Liu, Maximal loop method of automatic sketching of planar closed kinematic chains, Jixie Gongcheng Xuebao/J. Mech. Eng. 45 (11) (2009) 30-37.

[64] H. Ding, J. Zhao, Z. Huang, Unified structural synthesis of planar simple and multiple joint kinematic chains, Mech. Mach. Theory 45 (4) (2010) 555-568.

[65] M. Pucheta, N. Ulrich, A. Cardona, Combined graph layout algorithms for automated sketching of kinematic chains, ASME DE, vol. 4, 2012 , pp. 513-523.

[66] M. Pucheta, N. Ulrich, A. Cardona, Automated sketching of non-fractionated kinematic chains, Mech. Mach. Theory 68 (2013) 67-82.

[67] M. Saura, A. Celdran, D. Dopico, J. Cuadrado, Computational structural analysis of planar multibody systems with lower and higher kinematic pairs, Mech. Mach. Theory 71 (2014) 79-92.

[68] H.-S. Yan, Y.-T. Chiu, An improved algorithm for the construction of generalized kinematic chains, Mech. Mach. Theory 78 (2014) $229-247$.

[69] B. Paul, A unified criterion for the degree of constraint of plane kinematic chains, J. Appl. Mech. 27 (1) (1960) 196-200.

[70] N.I. Manolescu, For a united point of view in the study of the structural analysis of kinematic chains and mechanisms, J. Mech. 3 (3) (1968) 149-169.

[71] V.P. Agrawal, J.S. Rao, The mobility properties of kinematic chains, Mech. Mach. Theory 22 (5) (1987) 497-504.

[72] T.-L. Yang, F.-H. Yao, Topological characteristics and automatic generation of structural synthesis of spatial mechanisms. Part II. Automatic generation of structural types of kinematic chains, ASME DE, vol. 47, 1992, pp. 187-190.

[73] W. Li, Z. Wang, H. Li, Study on determining loops of planar kinematic chains, Proc. Inst. Mech. Eng. C J. Mech. Eng. Sci. 208 (1) (1994) 59-63.

[74] C. Tischler, A. Samuel, K. Hunt, Kinematic chains for robot hands - II. Kinematic constraints, classification, connectivity, and actuation, Mech. Mach. Theory 30 (8) (1995) 1217-1239.

[75] J. Yadav, C. Pratap, V. Agrawal, Freedom analysis of planar kinematic chains using the concept of distance, J. Mech. Des. Trans. ASME 118 (3) (1996) 367-371.

[76] M. Shoham, B. Roth, Connectivity in open and closed loop robotic mechanisms, Mech. Mach. Theory 32 (3) (1997) 279-293.

[77] N.P. Belfiore, Distributed databases for the development of mechanisms topology, Mech. Mach. Theory 35 (12) (2000) 1727-1744.

[78] N.P. Belfiore, A. Di Benedetto, Connectivity and redundancy in spatial robots, Int. J. Robot. Res. 19 (12) (2000) 1245-1261.

[79] S. Madan, R. Jain, Kinematic chains - isomorphism, inversions and degree of similarity using concept of connectivity, J. Inst. Eng. (India) Mech. Eng. Div. 82 (MAR) (2002) 164-169 (cited By, since 1996, 0).

[80] I. Popescu, D. Marghitu, E. Stoenescu, Kinematic chains with independent loops and spatial system groups, Arch. Appl. Mech. 75 (10-12) (2006) $739-754$.

[81] H. Ding, Z. Huang, A new theory for the topological structure analysis of kinematic chains and its applications, Mech. Mach. Theory 42 (10) (2007) $1264-1279$.

[82] D. Martins, A. Carboni, Variety and connectivity in kinematic chains, Mech. Mach. Theory 43 (10) (2008) 1236-1252.

[83] H. Ding, Z. Huang, D. Mu, Computer-aided structure decomposition theory of kinematic chains and its applications, Mech. Mach. Theory 43 (12) (2008) 1596-1609.

[84] H. Ding, J. Zhao, Z. Huang, The establishment of edge-based loop algebra theory of kinematic chains and its applications, Eng. Comput. 26 (2) (2010) 119-127.

[85] A. Liberati, N.P. Belfiore, A method for the identification of the connectivity in multi-loop kinematic chains: analysis of chains with total and partial mobility, Mech. Mach. Theory 41 (12) (2006) 1443-1466.

[86] S. Li, D. Wang, J. Dai, Topology of kinematic chains with loops and orientation of joints axes, Jixie Gongcheng Xuebao/J. Mech. Eng. 45 (6) (2009) $34-40$.

[87] G.S. Bedi, S. Sanyal, Joint connectivity: a new approach to detection of isomorphism and inversions of planar kinematic chains, J. Inst. Eng. (India) Mech. Eng. Div. 90 (January) (2010) 23-26.

[88] F. Freudenstein, An application of Boolean algebra to the motion of epicyclic drives, ASME J. Eng. Ind. 93 (1971) 176-182.

[89] F. Freudenstein, A. Yang, Kinematics and statics of a coupled epicyclic spur-gear train, Mech. Mach. Theory 7 (2) (1972) 263-275.

[90] E. Pennestrì, N.P. Belfiore, Kinematic and static force analysis of epicyclic gear trains, Conference on Appl. Mechanisms and Robotics, Cincinnati, OH, Vol. 1-6B, AMR, 1989.

[91] L.-W. Tsai, An application of Graph Theory to the detection of. fundamental circuits in epicyclic gear trains, in: IFToMM (Ed.), Ninth World Congress on the Theory of Machines and Mechanisms, vol. 1, 1995, pp. 692-696.

[92] J. Hopcroft, R. Tarjan, Efficient planarity testing, J. ACM 21 (4) (1974) 549-568.

[93] L.S. Woo, An algorithm for straight-line representation of simple planar graphs, J. Frankl. Inst. 287 (3) (1969) 197-284.

[94] G.N. Sandor, On the loop equations in kinematics, Proceedings of the Seventh Conference on Mechanisms, Penton Publishing, Cleveland, 1962.

[95] O. Shai, G. Pennock, Extension of Graph Theory to the duality between static systems and mechanisms, ASME J. Mech. Des. 128 (1) (2005) 179-191.

[96] J. Wittenburg, Dynamics of Systems of Rigid Bodies, Leitfäden der angewandten Mathematik und Mechanik, Teubner, 1977.

[97] G.C. Andrews, H.K. Kesavan, The vector-network model: a new approach to vector dynamics, Mech. Mach. Theory 10 (1) (1975) 57-75.

[98] R. Rogers, G. Andrews, Simulating planar systems using a simplified vector-network method, Mech. Mach. Theory 10 (6) (1975) 509-519.

[99] G.C. Andrews, J.R. Marc, R.J. Anderson, A general vector-network formulation for dynamic systems with kinematic constraints, Mech. Mach. Theory 23 (3) (1988) 243-256.

[100] M. Richard, R. Anderson, G. Andrews, The vector-network method for the modelling of mechanical systems, Math. Comput. Simul. 31 (6) (1990) 565-581.

[101] P.N. Sheth, J.J. Uicker, Imp (integrated mechanisms program), a computer-aided design analysis system for mechanisms and linkage, ASME 94 (1977) 454-464.

[102] J. Wittenburg, Dynamic of Systems of Rigid Bodies, Teubner, 1977.

[103] J.J. McPhee, M.G. Ishac, G.C. Andrews, Wittenburg's formulation of multibody dynamics equations from a graph-theoretic perspective, Mech. Mach. Theory 31 (2) (1996) 201-213.

[104] J. Polder, A Network Theory for Variable Epicyclic Gear Trains, Ph.D. Thesis Technische Hogeschool Delft, 1969.

[105] J. Wojnarowski, J. Kopeć, S. Zawślek, Gears and graphs, J. Theor. Appl. Mech. 44 (2006) 139-162.

[106] F. Freudenstein, A.T. Yang, Mechanics of epicyclic bevel-gear trains, ASME J. Eng. Ind. 95 (1973) 497-502. 
[107] G. Del Pio, E. Pennestrì, P.P. Valentini, Kinematic and power-flow analysis of bevel gears planetary gear trains with gyroscopic complexity, Mech. Mach. Theory 70 (2013) 523-537.

[108] E. Pennestrì, F. Freudenstein, Systematic approach to power flow and static-force analysis in epicyclic spur-gear trains, J. Mech. Des. Trans. ASME 115 (3) (1993) 639-644.

[109] T. Davies, Kirchhoff's circulation law applied to multi-loop kinematic chains, Mech. Mach. Theory 16 (3) (1981) $171-183$.

[110] E. Pennestrì, L. Mariti, P.P. Valentini, V.H. Mucino, Efficiency evaluation of gearboxes for parallel hybrid vehicles: theory and applications, Mech. Mach. Theory 49 (2012) 157-176.

[111] E. Pennestrì, F. Freudenstein, Mechanical efficiency of epicyclic gear trains, J. Mech. Des. Trans. ASME 115 (3) (1993) 645-651.

[112] L.W. Tsai, J.-J. Lee, Kinematic analysis of tendon-driven robotic mechanisms using Graph Theory, ASME J. Mech. 111 (PT3) (1989) 59-65.

[113] J. Dunlop, A. Khajepour, A new multi-terminal pulley model for use in graph theoretic modelling, Mech. Mach. Theory 35 (2000) $1601-1621$.

[114] M. Uyguroğlu, H. Demirel, Kinematic analysis of tendon-driven robotic mechanisms using oriented graphs, Acta Mech. 182 (3-4) (2006) $265-277$.

[115] J. Wojnarowski, A. Lidwin, The application of signal flow graphs - the kinematic analysis of planetary gear trains, Mech. Mach. Theory 10 (1) (1975) 17-31.

[116] L.W. Tsai, An algorithm for the kinematic analysis of epicyclic gear trains, Proc. of the Sixth Applied Mechanisms Conference, Kansas City, Missouri, 1985.

[117] D.G. Olson, D.R. Erdman, A.G. Riley, Topological analysis of single-degree-of-freedom planetary gear trains, Trends and Developments in Mechanisms, Machines, and Robotics, vol. 15, 1988, pp. 125-131.

[118] E. Pennestrì, N.P. Belfiore, Kinematic and static force analysis of epicyclic gear trains, Proc. 1st National Applied Mechanism and Robotics Conf., vol. I, University of Cincinnati, Cincinnati, OH, 1989.

[119] C.-H. Hsu, A graph notation for the kinematic analysis of differential gear trains, J. Frankl. Inst. 329 (5) (1992) $859-867$.

[120] C.-H. Hsu, K.-T. Lam, New graph representation for the automatic kinematic analysis of planetary spur-gear trains, J. Mech. Des. Trans. ASME 114 (1) (1992) $196-200$.

[121] A. Hedman, Transmission analysis - automatic derivation of relationships, J. Mech. Des. Trans. ASME 115 (4) (1993) $1031-1037$.

[122] C. Hsu, Displacement isomorphism of planetary gear trains, Mech. Mach. Theory 29 (1994) 513-523.

[123] S.Y.T. Lang, Graph-theoretic modelling of epicyclic gear systems, Mech. Mach. Theory 40 (5) (2005) 511-529.

[124] J. Drewniak, S. Zawiślack, Kinematic and dynamical analysis of closed kinematic chains using graphs and contour equations, Proc. Appl. Math. Mech. 9 (2009) 547-548.

[125] S. Drewniak, J. Zawiślack, Comparison of graph-based methods of kinematic analysis of planetary gears, Acta Mech. Autom. 4 (2010) 14-18.

[126] L.W. Tsai, G. Schultz, A motor-integrated parallel hybrid transmission, J. Mech. Des. Trans. ASME 126 (5) (2004) 889-894.

[127] H. Hsieh, L.W. Tsai, Kinematic analysis of epicyclic-type transmission mechanisms using the concept of fundamental geared entities, J. Mech. Des. Trans. ASME 118 (2) (1996) 294-299.

[128] S. Uematsu, An application of Graph Theory to the kinematic analysis of planetary gear trains, Int. J. Jpn Soc. Precis. Eng. 31 (2) (1997) $141-146$.

[129] E. Pennestrì, P.P. Valentini, F. Petti, Kinematic analysis of the antikythera gear mechanism by means of Graph Theory, DETC2002, vol. 4, 2002, pp. 387-394.

[130] E.L. Esmail, S.S. Hassan, An approach to power-flow and static force analysis in multi-input multi-output epicyclic-type transmission trains, J. Mech. Des. Trans. ASME 132 (1) (2010) 0110091-01100910.

[131] M. Raghavan, The analysis of planetary gear trains, ASME J. Mech. Robot. 2 (2010) 5.

[132] J.R. Gomà Ayats, U. Diego-Ayala, J. Minguella Canela, F. Fenollosa, J. Vivancos, Hypergraphs for the analysis of complex mechanisms comprising planetary gear trains and other variable or fixed transmissions, Mech. Mach. Theory 51 (2012) 217-229.

[133] D.R. Salgado, J.M. Del Castillo, Analysis of the transmission ratio and efficiency ranges of the four-, five-, and six-link planetary gear trains, Mech. Mach. Theory 73 (2014) 218-243.

[134] L.-W. Tsai, Kinematics of spatial robotic bevel-gear trains, IEEE J. Robot. Autom. 4 (2) (1988) 150-156.

[135] L.W. Tsai, C.C. Lin, The development of an atlas of bevel-gear-type spherical wrists mechanisms, Proc. 1st National Applied Mechanism and Robotics Conf., Vol. I, Cincinnati, $\mathrm{OH}, 1989$.

[136] E. Pennestrì, On the kinematic analysis of geared robotic wrists, Meccanica 26 (2-3) (1991) 155-160.

[137] R. Ma, K. Gupta, Signal flow graphs for spatial gear trains, ASME J. Mech. Des. 116 (1994) 326-331.

[138] N.P. Belfiore, E. Pennestrì, Characterization of kinematic and static performances of robotic geared wrists, Advances in Robot Kinematics and Computational Geometry, Kluwer Academic Publishers, Dordrecht, Olanda, 1994, pp. 209-218.

[139] N.P. Belfiore, E. Pennestrì, Kinematic and static torque analysis of remotely actuated geared wrists, in: L. Sciavicco, C. Bonivento, F. Nicolò (Eds.), Robot Control 1994 (SYROCO '94), 1994.

[140] M. Uyguroğlu, Y. Tokad, Kinematic analysis of robotic bevel-gear trains: an application of network model approach, Meccanica 33 (2) (1998) 177-194.

[141] M. Uyguroğlu, H. Demirel, Kinematic analysis of bevel-gear trains using graphs, Acta Mech. 177 (1-4) (2005) 19-27.

[142] S. Staicu, Inverse dynamics of a planetary gear train for robotics, Mech. Mach. Theory 43 (7) (2008) 918-927.

[143] I. Talpasanu, P. Simionescu, Kinematic analysis of epicyclic bevel gear trains with matroid method, ASME J. Mech. Des. 134 (2012) 8.

[144] J. Penaud, D. Alazard, A. Amiez, Kinematic analysis of spatial geared mechanisms, ASME J. Mech. Des. 134 (2012) 6.

[145] B. Yin, X. Li, Y. Chen, X. Guo, Y. Liang, Research on kinematics for planetary bevel-gear trains, Adv. Mater. Res. Vol. 479-481 (2012).

[146] I. Talpasanu, A general method for kinematic analysis of epicyclic bevel gear trains applied to robotic wrist mechanisms, ASME 2013 Int. Mech. Eng. Cong. and Exp., San Diego, CA, 2013.

[147] J. Wojnarowski, The graph method of determining the loads in complex gear trains, Mechanism 11 (2) (1976) $103-121$.

[148] J.W. Polder, J.B. Young, Lumped parameter model of planetary gear systems, Inst. Mech. Eng. (Lond.) Proc. 192 (1978) $251-258$.

[149] E. Pennestrì, N.P. Belfiore, R. Sinatra, A catalog of automotive transmissions with kinematic and power-flow analysis, Proc.Third Applied Mechanisms and Robotics Conference, No. Paper N. AMR-93-057, Cincinnati, OH, 1993.

[150] N.P. Belfiore, E. Pennestrì, R. Sinatra, Kinematic and power-flow analysis of epicyclic gear drives, MAPLETECH, Maple Tech. Newsl. 1 (1) (1994) 47-55

[151] L. Al-Hakim, A. Kusiak, J. Mathew, A graph-theoretic approach to conceptual design with functional perspectives, Comput. Aided Des. 32 (14) (2000) 867-875.

[152] C. Chen, J. Angeles, Virtual-power flow and mechanical gear-mesh power losses of epicyclic gear trains, ASME J. Mech. Des. 129 (2006) 107-113.

[153] N. Bucknor, Automatic determination of transmission powerflow mechanizability using Graph Theory, ASME 2007 Int. Des. Eng. Tech. Conf., Las Vegas, Nevada, 2007.

[154] C. Chen, Power flow analysis of compound epicyclic gear transmission: Simpson gear train, ASME J. Mech. Des. 133 (2011) 5.

[155] L.-W. Tsai, D.-Z. Chen, T.-W. Lin, Dynamic analysis of geared robotic mechanisms using Graph Theory, J. Mech. Des. Trans. ASME 120 (2) (1998) $240-244$.

[156] C.A. Nelson, R.J. Cipra, Simplified kinematic analysis of bevel epicyclic gear trains with application to power-flow and efficiency analyses, ASME J. Mech. 127 (2005) 278-286.

[157] L.P. Laus, H. Simas, D. Martins, Efficiency of gear trains determined using graph and screw theories, Mech. Mach. Theory 52 (2012) $296-325$.

[158] G. Mantriota, E. Pennestrì, Theoretical and experimental efficiency analysis of multi-degrees-of-freedom epicyclic gear trains, Multibody Syst. Dyn. 9 (2003) 389-408.

[159] E. Pennestrì, P.P. Valentini, A review of formulas for the mechanical efficiency analysis of two degrees-of-freedom epicyclic gear trains, J. Mech. Des. Trans. ASME 125 (3) (2003) 602-608.

[160] K.-T. Lam, A study on the mechanical efficiency of power split unit of the Toyota hybrid system, J. Sci. Innov. 1 (4) (2011) 7-16.

[161] A. Srinath, G. Yedukondalu, A. Jagadeesh, Mechanical efficiency of planetary gear trains: an estimate, Mech. Eng. Res. 1 (1) (2011) 97-102.

[162] C. Chen, T.T. Liang, Theoretic study of efficiency of two-DOFs of epicyclic gear transmission via virtual power, ASME J. Mech. Des. 133 (2011) 1-7.

[163] K. Davies, C. Chen, B. Chen, Complete efficiency analysis of epicyclic gear train with two degrees of freedom, ASME J. Mech. Des. 134 (2012) 8.

[164] M. Chen, Efficiency sensitivity of two-stage planetary gear transmission system based on Graph Theory, Appl. Mech. Mater. 215-216 (2012). 